\title{
Antibiotic Susceptibility and Molecular Characterization of Campylobacter jejuni Strain Isolated from a Guillain Barré Syndrome Child
}

\author{
Zobia Noreen $^{1} \cdot$ Mohammad Abrar $^{1} \cdot$ Fariha Siddiqui $^{1} \cdot$ Rani Faryal $^{2}$. \\ Haroon Hamid ${ }^{3}$ Habib Bokhari ${ }^{1}$
}

Received: 11 June 2015 / Accepted: 12 October 2015 / Published online: 12 November 2015

(C) Dr. K C Chaudhuri Foundation 2015

To the Editor : Campylobacter jejuni is among the leading etiological agents associated with bacterial diarrhea worldwide. In rare occasions, Campylobacter jejuni infection may trigger the human immune system to attack self-gangliosides resulting in Guillain-Barré syndrome [1]. We here, present case of a 7-yold girl suffering from acute flaccid paralysis of lower limbs after an episode of bloody diarrhea. The child was wheel chair bounded but did not have any difficulty in breathing or swallowing and did not receive any antibiotic treatment. The patient showed elevated levels of cerebrospinal fluid (CSF) (261 csf mg/dl). She was treated with $-5 \mathrm{~d}$ course of intravenous immunoglobulin (IVIG) at standard dose of $0.4 \mathrm{~g} / \mathrm{kg} / \mathrm{d}$. No ventilation support was needed. On day 25 th, she walked $-5 \mathrm{~m}$ distance with aid. C. jejuni was isolated from the patient's stool sample and identification was confirmed by using species specific (hipO) PCR [2]. The strain was found to be resistant to three antibiotics i.e., erythromycin, sulfamethoxazole + trimethoporim, cephalothin. PCR analysis for the detection of genes (sul1, sul2 and sul3) showed that the isolate was positive for all the three resistivity genes $[3$, 4]. Virulence genes i.e., cad $\mathrm{F}$ (fibronectin binding protein) and wla $\mathrm{N}$ (putative beta-1,3-galactosyl transferase) were present whereas $N e u A B$ (sialic acid biosynthesis gene) was absent [2]. The wla $\mathrm{N}$ gene in C. jejuni is proposed to encode an enzyme

Zobia Noreen and Mohammad Abrar contributed equally to this work.

Habib Bokhari

habib@comsats.edu.pk

1 Department of Biosciences, COMSATS Institute of Information Technology, Park Road, Chak Shahzad, Islamabad, Pakistan

2 Department of Microbiology, Quaid-i-Azam University, Islamabad, Pakistan

3 The Children's Hospital Lahore, Lahore, Pakistan $\beta$-1,3-galactosyl transferase that converts GM2-like LOS structure to a GM1-like structure. These GM1-like structures trigger the human host to produce the anti-GM1 antibodies which binds to GM1 expressed on the motor nerves of the limbs, resulting in Guillain-Barré syndrome [5]. The presence of wla $\mathrm{N}$ gene in the present isolate further confirms the role of the gene in triggering AMAN, a variant of GBS. To our knowledge this is the first case of GBS following infection with $C$. jejuni, reported from Pakistan. Further investigations on prevalence and molecular characterization of $C$. jejuni associated GBS may contribute to a better understanding of the disease.

\section{Conflict of Interest None.}

Source of Funding COMSATS Institute of Information Technology, Islamabad, Pakistan.

\section{References}

1. Perera VN, Nachamkin I, Ung H, et al. Molecular mimicry in Campylobacter jejuni: role of the lipo-oligosaccharide core oligosaccharide in inducing anti-ganglioside antibodies. FEMS Immunol Med Microbiol. 2007;50:27-36.

2. Fariha S, Akram M, Noreen N, Noreen Z, Bokhari H. Antibiotic susceptibility profiling and virulence potential of Campylobacter jejuni isolate from different sources in Pakistan. Asian Pac J Trop Med. 2015;8:197-202.

3. Kerrn MB, Klemmensen T, Frimodt-Mǿller N, Espersen F. Susceptibility of Danish Escherichia coli strains isolated from urinary tract infections and bacteraemia, and distribution of sul genes conferring sulphonamide resistance. J Antimicrob Chemother. 2002;50:513-6.

4. Perreten V, Boerlin P. A new sulfonamide resistance gene (sul3) in Escherichia coli is widespread in the pig population of Switzerland. Antimicrob Agents Chemother. 2003;47:1169-72.

5. Yuki N, Hartung H. Guillain-Barré syndrome. N Engl J Med. 2012;366:2294-304. 